\title{
The evolutionary psychology of hunger
}

\author{
Laith Al-Shawaf ${ }^{\mathrm{a}, \mathrm{b}, *}$ \\ a Department of Psychology, Bilkent University, Ankara, Turkey \\ ${ }^{\mathrm{b}}$ College of Life Sciences, Wissenschaftskolleg zu Berlin (Institute for Advanced Study), Germany
}

\section{A R T I C L E I N F O}

\section{Article history:}

Received 24 February 2016

Received in revised form 14 June 2016

Accepted 15 June 2016

Available online 18 June 2016

\section{Keywords:}

Evolutionary psychology

Hunger

Eating

Coordinating mechanisms

Superordinate mechanisms

\begin{abstract}
A B S T R A C T
An evolutionary psychological perspective suggests that emotions can be understood as coordinating mechanisms whose job is to regulate various psychological and physiological programs in the service of solving an adaptive problem. This paper suggests that it may also be fruitful to approach hunger from this coordinating mechanism perspective. To this end, I put forward an evolutionary task analysis of hunger, generating novel a priori hypotheses about the coordinating effects of hunger on psychological processes such as perception, attention, categorization, and memory. This approach appears empirically fruitful in that it yields a bounty of testable new hypotheses.
\end{abstract}

(C) 2016 Elsevier Ltd. All rights reserved.

\section{Theoretical background}

\subsection{Emotions as coordinating mechanisms}

An evolutionary psychological approach to the emotions suggests that they are coordinating mechanisms - informationprocessing programs that evolved to regulate the activity of other programs in the service of solving a specific adaptive problem (AlShawaf, Conroy-Beam, Asao, \& Buss, 2015; Cosmides \& Tooby, 2000; Tooby \& Cosmides, 1990; 2008). On this view, the emotions coordinate the activity of a wide range of psychological and physiological mechanisms, ranging from perception, attention, and memory to specialized inference mechanisms, physiology, and behavior.

This approach suggests that each emotion evolved to coordinate the operation of psychological and physiological mechanisms in solving a particular adaptive problem. For example, fear coordinates the activity of psychological and physiological programs in the service of avoiding predators or escaping danger (Bracha, 2004; Marks \& Nesse, 1994), disgust regulates mechanisms in order to avoid infection (Curtis, Aunger, \& Rabie, 2004; Tybur, Lieberman, Kurzban, \& DeScioli, 2012), and sexual arousal orchestrates programs in preparation for an advantageous sexual

\footnotetext{
* Department of Psychology, Bilkent University, Ankara, Turkey.

E-mail address: laith@bilkent.edu.tr.
}

opportunity (Al-Shawaf et al., 2015).

This coordinating mechanism approach was presented as a way of conceptualizing emotions (Cosmides \& Tooby, 2000; Tooby \& Cosmides, 1990; 2008), but it may also be usefully applied to drives or motivational states. Hunger is usually regarded as a motive or drive as opposed to an emotion (e.g., Kleinginna, Jr. \& Kleinginna, 1981; Lazarus, 1991; Oatley, Keltner, \& Jenkins, 2006), but it may be possible to use this approach to shed light on hunger and to generate novel hypotheses about how it coordinates other mechanisms in the body and mind. ${ }^{1}$ According to this view, hunger may usefully be regarded as a mechanism that coordinates the activity of psychological processes in the service of solving the adaptive problem of acquiring food (Cosmides \& Tooby, 2000).

In their presentation of the coordinating mechanism approach, Tooby and Cosmides (1990, 2008; Cosmides \& Tooby, 2000) suggested a list of 14 programs regulated by the emotions. I present these again here because they serve as a foundation for our hypothesis generation method: (1) perceptual mechanisms, (2) attention, (3) memory, (4) categorization, (5) motivational priorities, (6) current goals, (7) information-gathering adaptations, (8) specialized inference mechanisms, (9) communication \& expression, (10) learning mechanisms, (11) reflexes, (12) energy level,

\footnotetext{
${ }^{1}$ Indeed, the original authors of this idea suggest that, on their view, there may be no principled, non-arbitrary distinction between drive states and other emotion programs (Cosmides \& Tooby, 2000).
} 
mood, and effort allocation, (13) physiology, and (14) behavior.

The theoretical foundations of this approach, including a discussion of a) the conceptual strengths of this view, b) why mechanisms need coordination in the first place, and c) how to empirically test the validity of this approach, are all outlined in longer conceptual papers (Al-Shawaf et al., 2015; Cosmides \& Tooby, 2000; Tooby \& Cosmides, 1990; 2008). I therefore do not repeat those points here. Instead, in this article I approach hunger from this "coordinating mechanism" perspective, using a method called evolutionary task analysis to generate an array of novel $a$ priori hypotheses about hunger.

\subsection{Heuristic value and predictive power}

The coordinating mechanism approach offers a systematic hypothesis generation method based on evolutionary task analysis (Marr, 1982). It consists of several key questions: (1) what adaptive problem, if any, did this mechanism evolve to solve?, (2) which subtasks must be solved in the solution of this adaptive problem?, (3) which information-processing programs are capable of solving these subtasks?, and (4) how should these programs be coordinated to deliver a well-designed solution to this adaptive problem? (Al-Shawaf et al., 2015).

This framework provides a systematic way to generate novel empirical hypotheses for each candidate emotion or motivational drive by asking which programs it is expected to coordinate and how it is expected to do so. Of course, not every coordinating mechanism is expected to regulate the full list of 14 programs listed above.

In what follows, I apply this hypothesis-generation framework to hunger. The purpose of this exercise is not to provide a comprehensive analysis of hunger or generate an exhaustive list of hypotheses, but rather to illustrate the heuristic value and predictive power of this approach and to suggest that there may be utility in conceptualizing hunger as a coordinating mechanism.

\section{Novel hypotheses}

\subsection{Motivational priorities}

Hunger, a universal mechanism that evolved to solve a critical adaptive problem, is the first step in the food cycle - the sequence of behaviors that culminates in eating (Rozin \& Todd, 2015). In order to solve the problem of food acquisition, hunger likely reorders an individual's motivational priorities, elevating the importance of finding food and temporarily inhibiting other concerns such as status enhancement, mating opportunities, and pathogen avoidance. The intensity of this motivational reordering should depend on the intensity of the hunger as well as the immediacy and importance of other adaptive problems that the organism is facing. For example, when simultaneously presented with hunger and a threat or opportunity of great importance to fitness, hunger may have a more muted effect on motivational reordering. In extreme cases, intense hunger may be suppressed in order to solve a more pressing adaptive problem, such as urgent predator avoidance or the consummation of a valuable mating opportunity. We can refer to the simultaneous presentation of conflicting adaptive problems as an adaptive metaproblem.

Solving an adaptive metaproblem requires a tradeoff between the mechanisms responsible for solving the competing adaptive problems. In some cases, one adaptive problem may dominate, as when hunger is suppressed during a near-death escape from a predator. In other cases, a compromise solution is possible, and an individual may simultaneously allocate a portion of her resources to two different adaptive problems (see Crabbe, 2007; Prescott,
Bryson, \& Seth, 2007; and Redgrave, Prescott, \& Gurney, 1999, for related discussions about natural action selection mechanisms).

Evolutionary psychological reasoning suggests that the solutions to these adaptive metaproblems should differ systematically by sex, context, and individual difference variables. For example, given men's stronger predilection for short-term mating and casual sex (Buss, 2003; Lippa, 2009; Symons, 1979), a short-term mating opportunity with an attractive member of the opposite sex should suppress hunger much more powerfully among men than among women. By contrast, given the robust sex difference in disgust (women are more easily disgusted than men; Al-Shawaf \& Lewis, 2013; Al-Shawaf, Lewis, \& Buss, 2014; Curtis et al., 2004; Tybur et al., 2012), the presentation of a pathogen threat should suppress hunger more effectively among women than among men.

Ultimately, the outcome of tradeoffs between competing mechanisms such as hunger and sexual arousal will depend on the costs and benefits associated with allocating resources to one adaptive problem over another. Ancestrally, the costs and benefits of solving one adaptive problem over another would often have differed systematically as a function of a) context, b) sex, and c) individual difference variables such as immune competence, mate value, and ability to evade predators.

These systematic differences in costs and benefits between different contexts and individuals can be used to generate a priori hypotheses about a) how hunger is expected to coordinate mechanisms in a context-specific and individual-specific manner, as well as b) how hunger is expected to trade off with competing drives or emotions when an organism is faced with distinct adaptive problems at once. More broadly, this coordinating mechanism approach offers a way to generate context-specific hypotheses about how hunger might regulate mechanisms in the body and mind.

\subsection{Perception}

Hunger may influence perceptual mechanisms in order to solve the adaptive problem of finding food. Early theorists recognized this possibility: "What we must study ... are the variations perception itself undergoes when one is hungry, in love, in pain, or solving a problem" (Bruner \& Goodman, 1947, p. 33).

These effects may operate through more than one perceptual mode. For example, there is some evidence that hunger leads to more positive evaluations of flavor, motivating further eating when an individual has yet to reach satiation (e.g., Cabanac, 1971). We might hypothesize that the gustatory reward associated with eating should be directly dependent on hunger level, with greater pleasure accompanying greater hunger and diminishing hedonic returns accompanying increased satiety. Tying gustatory pleasure to metabolic need in this way would be a good design for a system that motivates eating.

There is already evidence to suggest that food odors - but not other odors - are more pleasant when nutritionally depleted (Duclaux, Feisthauer, \& Cabanac, 1973). We also have evidence that hunger increases the perceived brightness of foods (Gilchrist \& Nesberg, 1952), and improves perceptual encoding or accuracy of food-relevant stimuli (Lazarus, 1998; Radel \& Clément-Guillotin, 2012), and that thirst may increase the perceptual or cognitive accessibility of drinking-related stimuli (Aarts, Dijksterhuis, \& De Vries, 2001), as well as making it easier for people to perceive transparency (a quality of water) in ambiguous stimuli (Changizi \& Hall, 2001). It would also be reasonable to expect that hunger and thirst make food and drink look more visually appealing as well (but see Hoefling et al., 2009).

Relatedly, hunger may enhance olfactory acuity, aiding the process of detecting and locating food when nutritionally depleted. This modulation of olfactory sensitivity by metabolic status and 
feeding state has been demonstrated in nonhuman species such as rodents, Drosophila, and C. elegans (Fadool, Tucker, \& Pedarzani, 2011; Palouzier-Paulignan et al., 2012; Root, Ko, Jafari, \& Wang, 2011). Testing this hypothesis in humans seems both feasible and worthwhile.

An intriguing further possibility is that hunger may improve detection of food-related sounds (e.g., sounds emitted by prey animals, or the sound of group members eating nearby). These hypotheses about olfactory and auditory perception can be tested in the lab by experimentally manipulating hunger and then presenting audio recordings and odors of different kinds, testing participants' accuracy and sensitivity in detecting food-relevant and foodirrelevant sounds and smells.

\subsection{Attention and problem solving}

Hunger may influence attention, too, narrowing an organism's attentional span and sharpening its focus to food and food-relevant stimuli (Channon \& Hayward, 1990; Mogg, Bradley, Hyare, \& Lee, 1998; Stockburger, Schmälzle, Flaisch, Bublatzky, \& Schupp, 2009). To deliver a well-designed solution to this adaptive problem, hunger must down-regulate attention to other stimuli that would normally capture an organism's interest. Severe hunger may even suppress attention to fitness-relevant stimuli such as pathogens, attractive mates, or valuable alliance-building opportunities (but see Ainsworth \& Maner, 2014).

It is also possible to generate very specific a priori predictions about the effects of context and individual differences. For example, hunger's ability to shift attention away from pathogens and toward food should be attenuated in people who are immunocompromised. Hunger's ability to suppress attention to other stimuli of adaptive significance should also depend on the magnitude of the fitness threat or opportunity posed by those other stimuli. For example, hunger should be more effective at shifting a person's attention away from an unattractive mate or a moderately dangerous predator compared to a highly attractive mate or an extremely dangerous predator.

It may also be difficult for hungry individuals to sustain attention on food-irrelevant tasks. For example, hungry people may find it hard to engage in future planning, problem solving, and extended conversations. There should be a striking exception for foodacquisition problems, which we might predict will elicit sustained interest and attention from hungry individuals. I would therefore hypothesize that hunger has opposite effects on different kinds of problem solving: it leads to a decrement in people's problemsolving abilities in most domains, yet simultaneously enhances their ability to solve food-acquisition problems.

We can test these predicted effects of hunger on attention and problem solving using problem-solving tasks that vary in hungerrelevance, dot-probe tasks, fMRI studies, and eye-tracking methods (e.g., Mogg et al., 1998; Nijs, Muris, Euser, \& Franken, 2010; Siep et al., 2009). As with many of the mechanisms discussed in this paper, the extent of hunger's regulatory effects on attention should depend on the intensity of the hunger.

\subsection{Categorization and conceptual frameworks}

Just as fear automatically imposes conceptual frameworks that cause us to view our surroundings in terms of safety and danger (Tooby \& Cosmides, 1990), hunger may automatically impose conceptual frameworks and categorization schemes relevant to the adaptive problem of finding food. For example, at the most global level, hunger may lead organisms to categorize items as either food or not food, with different implications for approach behavior. Within the category of food, items may be further categorized as safe or spoiled. And nested in the category of safe foods, hunger may further categorize items according to caloric density or nutritional value. Higher priorities should be assigned to foods of greater value and foods containing specific nutrients that an organism lacks (e.g., Rozin \& Vollmecke, 1986; Rozin \& Todd, 2015; Siep et al., 2009).

Evolutionary thinking suggests that this categorization may be adaptively biased, such that intense hunger results in a more lenient categorization of safe foods (e.g., see Cabanac, 1971). In the extreme, we might expect an organism on the brink of starvation to categorize as "safe" food sources that would otherwise be classified as spoiled, as long as the on-average cost of such overinclusive categorization is less than the cost of forgoing viable food sources and risking death by starvation.

Extreme hunger should also suppress disgust to potentially pathogenic foods (Hoefling et al., 2009). As individuals approach starvation, we should see a muted disgust response to foods that would otherwise trigger revulsion. If this response is an adaptation against starvation, it should be highly specific to food, and not other disgusting items. Evolutionary considerations therefore suggest that hunger should have no effect on disgust toward open sores, bodily effluvia, or other sources of pathogens and parasites. This appears to be exactly the pattern of results we see (Hoefling et al., 2009).

Importantly, severe food shortages were likely more common among our hunter-gatherer ancestors than they are in today's industrialized nations, and hunger probably played a prominent role in regulating psychology and behavior during human evolution. It is easy to underestimate the significance of this psychophysiological state in affluent modern-day societies, but hunger remains a pervasive aspect of hundreds of millions of people's lives in developing nations and in modern hunter-gatherer societies (Gat, 2000; Gurven, Hill, Kaplan, Hurtado, \& Lyles, 2000; Sanchez \& Swaminathan, 2005; Serageldin, 2002; Speth \& Spielmann, 1983), and was likely a recurrent condition during the evolution of our species.

In addition to affecting the way we categorize foods and pathogens, hunger may also affect the way we categorize other humans. For example, hunger may lead us to categorize others as either having or not having surplus food that they might be willing to share. Similarly, hungry individuals may temporarily categorize others in terms of whether they are likely to be useful in helping with food acquisition (e.g., as a hunting or foraging partner).

The key point is that we may expect hunger to impose conceptual frameworks and categorization schemes relevant to the problem of securing food. We might therefore expect hunger to cause people to adaptively categorize objects, other people, and events in ways that facilitate the acquisition of food.

\subsection{Memory}

An intriguing possibility is that hunger might impact memory mechanisms in the service of acquiring food. For example, hunger may increase the accessibility of memories specifying locations in which food was successfully obtained in the past. Indirect support for this hypothesis comes from fMRI evidence that hunger preferentially enhances recognition memory for food over nonfood (Morris \& Dolan, 2001), and from evidence that people in a field setting show preferential recall for the locations of calorie-dense food items over those of calorie-sparse ones (New, Krasnow, Truxaw, \& Gaulin, 2007; Krasnow et al., 2011). Additional evidence that hunger might impact memory comes from research showing superior word sequence recall for words directly following food-related words, but only among hungry individuals (see Lerner, Singer, \& Triandis, 1958). During times of nutritional scarcity, hunger may trigger especially strong encoding of information that 
can be used to resolve current or future hunger. Such a mechanism might encode locations where food is available, useful methods of acquiring or preserving food, or individuals who offer food during one's time of metabolic need. Intriguing indirect evidence for this sort of hunger-mediated memory comes from studies showing that Drosophila flies "must be hungry to form and retrieve appetitive memory" (Plaçais \& Preat, 2013, p.440).

We might also speculate that hunger could affect an individual's memory for group members. For example, hunger may temporarily increase the accessibility of memories indexing who typically shares food and who is stingy with it, who was recently observed to have surplus food, and who owes one an outstanding favor that might be repaid in the currency of food. These hypotheses are preliminary, but they represent an initial foray into the ways that hunger might regulate cognitive processes in the service of food acquisition. And they are testable.

\subsection{Eating, the deactivation of hunger, and mechanism "offlining"}

Eating marks the solution of the adaptive problem that hunger was designed to solve. Eating should therefore be a critical stopping point, terminating the coordinating effects of hunger on other mechanisms (assuming that one eats enough to sate general hunger plus any nutrient-specific hungers). This suggests the hypothesis that eating triggers a relatively abrupt deactivation of hunger's coordinating effects on other mechanisms. This should manifest itself in large pre-eating to post-eating shifts in people's perception, attention, categorization, memory, and motivational priorities.

For example, this hypothesis suggests that after sating themselves, people's motivational priorities are once again reordered, attention is broadened beyond food-related stimuli, memories of other duties and responsibilities resurface, categorization is no longer dominated by food, and previously suppressed adaptive problems such as mating and pathogen avoidance come to the fore again. For example, the sight and smell of more food can become disgusting after one is sated, a stark reversal in hunger's disgustsuppression effects. We might therefore expect that eating, a discrete event that ushers in a suite of psychophysiological changes (Liu, Gao, \& Fox, 2000), annuls or reverses the effects of hunger and marks the endpoint of the motivational state. ${ }^{2}$ This hypothesis suggests that we should see stark differences in people's attention, perception, categorization, and memory before and after eating. An intriguing hint of this possibility comes from a memory study: "Most notably, in the 20 min interval between pre-satiation and post-satiation scans, a $>20 \%$ memory advantage for food stimuli relative to non-food was abolished in all 10 subjects" (Morris \& Dolan, p. 5307).

Of course, the effects of eating cannot be instantaneous. It takes time for hunger to dissipate (e.g., Liu, Gao, Liu, \& Fox, 2000; de Graaf, Blom, Smeets, Stafleu, \& Hendriks, 2004), so the return to baseline triggered by a meal may be speedy but not immediate. This return to baseline activity may be more pressing for certain mechanisms than for others. Stated differently, different sequences of mechanism "offlining" may have different impacts on fitness. During the evolution of human hunger mechanisms, designs that coordinated this "offlining" in a systematic and beneficial manner would have outcompeted designs that turned programs off randomly or in inappropriate sequence.

This suggests the hypothesis that eating reverts mechanisms to

\footnotetext{
2 This is admittedly an oversimplification. Eating is not a discrete event, but rather a continuous process whose duration can vary. The "dissolving" effect of eating may therefore itself be continuous, and should depend on whether the organism has eaten enough to sate itself.
}

baseline activity in an orderly and predictable sequence, with more urgent and more impactful changes taking precedence. For example, the refocusing of attention on rivals or predators and the reordering of motivational priorities may be more pressing than the negation of hunger's effects on memory. If this hypothesis is correct, we should expect to see broadened attentional focus and renewed pathogen disgust before observing the reemergence of memories of other duties and responsibilities. The broader point is that it is reasonable to expect the dissipation of hunger to systematically deactivate mechanisms in a predictable sequence according to the urgency or importance of the changes.

At present, these suggestions are still speculative. But they highlight the fact that an evolutionary psychological approach points to a new conceptualization of hunger, novel hypotheses about both hunger and eating, and new ideas about how eating may terminate the coordinating effects of hunger in a systematic and predictable manner. Whatever its theoretical advantages, this perspective is empirically fruitful in the sense that it provides a method for systematically generating a priori hypotheses about hunger, as well as other drives and emotions.

This approach also provides a logical way to generate hypotheses about the "stopping points" of a drive or an emotion. Whenever a discrete event marks the solution to an adaptive problem that a motivational drive evolved to solve, that event can be expected to cause the drive to dissipate. Consequently, the event in question should dissolve the coordinating effects of the drive, resulting in a pronounced pre-event to post-event shift in a variety of mechanisms ranging from memory to attention to motivational priorities. We have hypothesized that in the case of sexual arousal, this "dissolving" event is orgasm (for males more strongly than females; Al-Shawaf et al., 2015). In the case of hunger, this "dissolving" event is eating. This leads to the testable hypothesis that eating until sated will cause the relatively rapid dissolution of hunger's effects on attention, perception, memory, and other psychological processes described above.

\section{Conclusions}

An evolutionary psychological approach suggests that the emotions are coordinating mechanisms whose evolved function is to orchestrate the activity of other mechanisms in the service of solving an adaptive problem (Al-Shawaf et al., 2015; Cosmides \& Tooby, 2000; Tooby \& Cosmides, 2008). For example, disgust coordinates mechanisms in the service of avoiding parasitic infection (Curtis et al., 2004; Tybur et al., 2012), fear regulates programs to escape from danger (Bracha, 2004; Marks \& Nesse, 1994; Marks, 1987), and romantic love coordinates mechanisms in the service of forming a long-term mating bond (Buss, 2006).

This approach might also be usefully applied to states such as hunger, pain, and sexual arousal. These states are not traditionally regarded as emotions, but may nonetheless be fruitfully regarded as coordinating mechanisms that have disparate effects on perception, attention, memory, conceptual categorization, and other psychological processes.

Empirically, this approach provides a powerful hypothesisgeneration method. Researchers can use evolutionary task analysis to systematically generate an array of novel hypotheses for any coordinating mechanism - including hunger - by asking (1) what adaptive problem, if any, the mechanism might have evolved to solve, (2) what subtasks constitute the adaptive problem, (3) which psychological mechanisms are capable of solving these subtasks, and (4) how these mechanisms ought to be coordinated to produce a well-designed solution to the adaptive problem. This systematic approach can be applied to any candidate emotion or drive, producing a priori hypotheses about the effects of the mechanism on 
psychological programs, the sequence of these effects, the contextsensitivity of these effects, and even the coordinated dissolution of these effects. Evolutionary task analysis thus offers both heuristic value and predictive power, and may be used to great effect in the study of emotions and motivational drives.More broadly, whatever its theoretical merits, this "coordinating mechanism" approach raises new questions, suggests new domains of inquiry, and appears to be empirically fruitful (Al-Shawaf et al., 2015; Cosmides \& Tooby, 2000; Tooby \& Cosmides, 2008). The next step will be to test some of the hypotheses in this paper and see how they stand up to empirical investigation.

\section{Acknowledgements}

The author wishes to thank Peter M. Todd and an anonymous reviewer for their helpful comments on an earlier draft of this paper, as well as Dan Conroy-Beam for our early discussions about adaptive metaproblems.

\section{References}

Aarts, H., Dijksterhuis, A., \& Vries, P. (2001). On the psychology of drinking: Being thirsty and perceptually ready. British Journal of Psychology, 92(4), 631-642.

Ainsworth, S. E., \& Maner, J. K. (2014). Hunger moderates the activation of psychological disease avoidance mechanisms. Evolutionary Behavioral Sciences, 8(4), 303-313.

Al-Shawaf, L., Conroy-Beam, D., Asao, K., \& Buss, D. M. (2015). Human emotions: An evolutionary psychological perspective. Emotion Review, 1-14. http://dx.doi.org/ $10.1177 / 1754073914565518$.

Al-Shawaf, L., \& Lewis, D. M. G. (2013). Exposed intestines and contaminated cooks: Sex, stress, \& satiation predict disgust sensitivity. Personality and Individual Differences, 54, 698-702.

Al-Shawaf, L., Lewis, D. M., \& Buss, D. M. (2014). Disgust and mating strategy. Evolution and Human Behavior, 36(3), 199-205. http://dx.doi.org/10.1016 j.evolhumbehav.2014.11.003.

Bracha, H. S. (2004). Freeze, flight, fight, fright, faint: Adaptationist perspectives on the acute stress response spectrum. CNS Spectrums, 9(9), 679-685.

Bruner, J. S., \& Goodman, C. C. (1947). Value and need as organizing factors in perception. The Journal of Abnormal and Social Psychology, 42(1), 33-44.

Buss, D. M. (2003). The evolution of desire: Strategies of human mating. New York: Basic Books.

Buss, D. M. (2006). The evolution of love. In R. J. Sternberg, \& K. Weis (Eds.), The new psychology of love (pp. 65-86). Yale: University Press.

Cabanac, M. (1971). Physiological role of pleasure. Science, 173(4002), 1103-1107.

Changizi, M. A., \& Hall, W. G. (2001). Thirst modulates a perception. Perception, 30(12), 1489-1497.

Channon, S., \& Hayward, A. (1990). The effect of short-term fasting on processing of food cues in normal subjects. International Journal of Eating Disorders, 9(4), $447-452$.

Cosmides, L., \& Tooby, J. (2000). Evolutionary psychology and the emotions. In M. Lewis, \& J. M. Haviland-Jones (Eds.), Handbook of emotions (2nd ed., pp. 91-115). New York: Guilford.

Crabbe, F. L. (2007). Compromise strategies for action selection. Philosophical Transactions of the Royal Society of London B: Biological Sciences, 362(1485), 1559-1571.

Curtis, V., Aunger, R., \& Rabie, T. (2004). Evidence that disgust evolved to protect from risk of disease. Proceedings of the Royal Society B, 271, 131-133.

Duclaux, R., Feisthauer, J., \& Cabanac, M. (1973). Effects of a meal on the pleasantness of food and nonfood odors in man. Physiology \& Behavior, 10(6), 1029-1033.

Fadool, D. A., Tucker, K., \& Pedarzani, P. (2011). Mitral cells of the olfactory bulb perform metabolic sensing and are disrupted by obesity at the level of the Kv1. 3 ion channel. PLoS One, 6(9), e24921.

Gat, A. (2000). The human motivational complex: Evolutionary theory and the causes of hunter-gatherer fighting. Part I. Primary somatic and reproductive causes. Anthropological Quarterly, 20-34.

Gilchrist, J. C., \& Nesberg, L. S. (1952). Need and perceptual change in need-related objects. Journal of Experimental Psychology, 44(6), 369-376.

de Graaf, C., Blom, W. A., Smeets, P. A., Stafleu, A., \& Hendriks, H. F. (2004). Biomarkers of satiation and satiety. The American Journal of Clinical Nutrition, 79(6), 946-961.

Gurven, M., Hill, K., Kaplan, H., Hurtado, A., \& Lyles, R. (2000). Food transfers among Hiwi foragers of Venezuela: Tests of reciprocity. Human Ecology, 28(2), 171-218.

Hoefling, A., Likowski, K. U., Deutsch, R., Häfner, M., Seibt, B., Mühlberger, A., et al. (2009). When hunger finds no fault with moldy corn: Food deprivation reduces food-related disgust. Emotion, 9(1), 50-58.

Kleinginna, P. R., Jr., \& Kleinginna, A. M. (1981). A categorized list of emotion definitions, with suggestions for a consensual definition. Motivation and Emotion, 5(4), 345-379.

Krasnow, M. M., Truxaw, D., Gaulin, S. J., New, J., Ozono, H., Uono, S., et al. (2011). Cognitive adaptations for gathering-related navigation in humans. Evolution and Human Behavior, 32(1), 1-12.

Lazarus, R. S. (1991). Emotion and adaptation. New York: Oxford University Press.

Lazarus, R. S. (1998). Fifty years of the research and theory of R.S. Lazarus: An analysis of historical and perennial issues. Mahwah, New Jersey: Lawrence Erlbaum Associates.

Lerner, R. G., Singer, I., \& Triandis, H. C. (1958). Serial verbal learning under two conditions of hunger motivation. Journal of Experimental Psychology, 55(6), $572-574$.

Lippa, R. A. (2009). Sex differences in sex drive, sociosexuality, and height across 53 nations: Testing evolutionary and social structural theories. Archives of Sexual Behavior, 38, 631-651.

Liu, Y., Gao, J., Liu, H., \& Fox, P. (2000). The temporal response of the brain after eating revealed by functional MRI. Nature, 405, 1058-1062.

Marks, I. M. (1987). Fears, phobias, and rituals: Panic, anxiety, and their disorders. New York: Oxford University Press.

Marks, I. M., \& Nesse, R. M. (1994). Fear and fitness: An evolutionary analysis of anxiety disorders. Ethology and Sociobiology, 15(5), 247-261.

Marr, D. (1982). Vision: A computational investigation into the human representation and processing of visual information. San Francisco: Freeman.

Mogg, K., Bradley, B. P., Hyare, H., \& Lee, S. (1998). Selective attention to food-related stimuli in hunger: Are attentional biases specific to emotional and psychopathological states, or are they also found in normal drive states? Behaviour Research and Therapy, 36(2), 227-237.

Morris, J. S., \& Dolan, R. J. (2001). Involvement of human amygdala and orbitofrontal cortex in hunger-enhanced memory for food stimuli. The Journal of Neuroscience, 21(14), 5304-5310.

New, J., Krasnow, M. M., Truxaw, D., \& Gaulin, S. J. (2007). Spatial adaptations for plant foraging: Women excel and calories count. Proceedings of the Royal Society of London B: Biological Sciences, 274(1626), 2679-2684.

Nijs, I. M., Muris, P., Euser, A. S., \& Franken, I. H. (2010). Differences in attention to food and food intake between overweight/obese and normal-weight females under conditions of hunger and satiety. Appetite, 54(2), 243-254.

Oatley, K., Keltner, D., \& Jenkins, J. M. (2006). Understanding emotions ( $2^{\text {nd }}$ ed.). Malden, MA: Blackwell.

Palouzier-Paulignan, B., Lacroix, M. C., Aimé, P., Baly, C., Caillol, M., Congar, P., et al. (2012). Olfaction under metabolic influences. Chemical Senses, 37(9), 769-797.

Plaçais, P. Y., \& Preat, T. (2013). To favor survival under food shortage, the brain disables costly memory. Science, 339(6118), 440-442.

Prescott, T. J., Bryson, J. J., \& Seth, A. K. (2007). Introduction. Modelling natural action selection. Philosophical Transactions of the Royal Society of London B: Biological Sciences, 362(1485), 1521-1529.

Radel, R., \& Clément-Guillotin, C. (2012). Evidence of motivational influences in early visual perception: Hunger modulates conscious access. Psychological Science, 23(3), 232-234.

Redgrave, P., Prescott, T. J., \& Gurney, K. (1999). The basal ganglia: A vertebrate solution to the selection problem? Neuroscience, 89(4), 1009-1023.

Root, C. M., Ko, K. I., Jafari, A., \& Wang, J. W. (2011). Presynaptic facilitation by neuropeptide signaling mediates odor-driven food search. Cell, 145(1), 133-144.

Rozin, P., \& Todd, P. M. (2015). The evolutionary psychology of food intake and choice. In D. M. Buss (Ed.), The handbook of evolutionary psychology (2nd ed., pp. 183-205). Wiley.

Rozin, P., \& Vollmecke, T. A. (1986). Food likes and dislikes. Annual Review of Nutrition, 6(1), 433-456.

Sanchez, P. A., \& Swaminathan, M. S. (2005). Cutting world hunger in half. Science, 307(5708), 357-359.

Serageldin, I. (2002). World poverty and hunger: The challenge for science. Science, 296(5565), 54-58

Siep, N., Roefs, A., Roebroeck, A., Havermans, R., Bonte, M. L., \& Jansen, A. (2009). Hunger is the best spice: An fMRI study of the effects of attention, hunger and calorie content on food reward processing in the amygdala and orbitofrontal cortex. Behavioural Brain Research, 198(1), 149-158.

Speth, J. D., \& Spielmann, K. A. (1983). Energy source, protein metabolism, and hunter-gatherer subsistence strategies. Journal of Anthropological Archaeology, 2(1), $1-31$.

Stockburger, J., Schmälzle, R., Flaisch, T., Bublatzky, F., \& Schupp, H. (2009). The impact of hunger on food cue processing: An event-related brain potential study. Neuroimage, 47(4), 1819-1829.

Symons, D. (1979). The evolution of human sexuality. New York: Oxford University Press.

Tooby, J., \& Cosmides, L. (1990). The past explains the present: Emotional adaptations and the structure of ancestral environments. Ethology and Sociohiology, 11, $375-424$.

Tooby, J., \& Cosmides, L. (2008). The evolutionary psychology of the emotions and their relationship to internal regulatory variables. In M. Lewis, J. M. HavilandJones, \& L. F. Barrett (Eds.), Handbook of emotions (3rd ed., pp. 114-137). New York: Guilford.

Tybur, J. M., Lieberman, D., Kurzban, R., \& DeScioli, P. (2012). Disgust: Evolved function and structure. Psychological Review, 120(1), 65-84. 